Third Meeting, January 8, 1892.

John Alison, Esq., M.A., F.R.S.E., Vice-President, in the Chair.

On the smallest number of entries necessary in a table of logarithms to seven decimal places.

By Professor J. E. A. Stegand.

Taking seven figure logarithms it is required to find at what interval from $n$, a number of five figures, the next entry need be made, in order that any intermediate logarithm may be calculated by the method of proportional parts.

We have

$$
\log (n+d)-\log n=\mu\left\{\frac{d}{n}-\frac{d^{2}}{2 n^{2}}+\ldots\right\} \quad \ldots \quad \ldots
$$

and

$$
\log (n+x)-\log n=\mu\left\{\frac{x}{n}-\frac{x^{2}}{2 n^{2}}+\ldots\right\} \quad \ldots \quad \ldots
$$

also

$$
\frac{x}{d}\{\log (n+d)-\log n\}=\mu\left\{\frac{x}{n}-\frac{x d}{2 n^{2}}+\ldots\right\} .
$$

This latter quantity, which we add to $\log n$ in order to find $\log$ $(n+x)$ approximately, is got by calculation, and its error (in defect) from the true quantity, $(2)$, is

$$
\mu x\left\{\frac{(d-x)}{2 n^{2}}-\frac{\left(d^{2}-x^{2}\right)}{3 n^{3}}+\ldots\right\} ;
$$

the greatest value of this error is when $x=\frac{1}{2} d$, and it then is $\mu d^{2} / 8 n^{2}$.

In order that there may not be an error of a unit in the seventh place we must have $\mu d^{2} / 8 n^{2}<\cdot 0000001$.

Whence

$$
\begin{aligned}
& d / n<\sqrt{.00000185} \\
& \text { or } d<.00136 n \text {, }
\end{aligned}
$$

and varies from $13 \cdot 6$ to 136 in the ordinary course of the tables. 
For example, taking one of the most unfavourable cases

$$
\begin{aligned}
& \log 99864=4.9994090 \\
& \log 100000=5.0000000
\end{aligned}
$$

and therefore

$\log 99932=4.9997045$

which is correct to within a unit in the last figure.

In fact, the number of entries really required in a table is given by

$$
\begin{aligned}
(1.00136)^{n} & =10, \\
n & =1 / .00059024 \\
& =\text { about } 1700,
\end{aligned}
$$

\begin{tabular}{|c|c|c|c|c|c|c|c|}
\hline 10000 & to 20000 & every & tenth & number & $\ldots$ & 1000 & entrie \\
\hline 20000 & „30000 & $\ldots$ & twentieth & $\ldots$ & $\ldots$ & 500 & , \\
\hline 30000 & " 40000 & $\ldots$ & fortieth & ... & $\ldots$ & 250 & " \\
\hline 40000 & , 80000 & $\ldots$ & fiftieth & $\ldots$ & $\ldots$ & 800 & " \\
\hline 80000 &, 100000 & $\ldots$ & hundredth & $\ldots$ & $\ldots$ & 200 & ", \\
\hline & & & & & & $\overline{2750}$ & " \\
\hline
\end{tabular}

whence

instead of 90000 .

The table might be arranged

If we apply the same method to a four figure table, all we require is that

or

$$
\begin{aligned}
& \mu d^{2} / 8 n^{2}<.0001, \\
& d< \sqrt{.0019 n}, \\
& d<\quad .044 n .
\end{aligned}
$$

Thus to obtain any logarithm to 4 decimal places we may, for example, work with only the logarithms of

$\begin{array}{lllllllll}104 & 135 & 170 & 208 & 270 & \mathbf{3 4 8} & \mathbf{4 4 8} & \mathbf{5 8 0} & \mathbf{7 6 0} \\ 108 & 140 & 175 & \mathbf{2 1 6} & 280 & \mathbf{3 6 0} & \mathbf{4 6 4} & 600 & \mathbf{7 9 0} \\ 112 & 145 & 180 & \mathbf{2 2 4} & 290 & \mathbf{3 7 5} & \mathbf{4 8 0} & \mathbf{6 2 5} & \mathbf{8 0 0} \\ 116 & 150 & 185 & \mathbf{2 3 2} & \mathbf{3 0 0} & \mathbf{3 9 0} & \mathbf{5 0 0} & \mathbf{6 5 0} & \mathbf{8 3 0} \\ 1,20 & 155 & 190 & \mathbf{2 4 0} & \mathbf{3 1 2} & 400 & \mathbf{5 2 0} & \mathbf{6 7 5} & \mathbf{8 6 5} \\ 125 & 160 & 195 & \mathbf{2 5 0} & \mathbf{3 2 4} & \mathbf{4 1 6} & \mathbf{5 4 0} & \mathbf{7 0 0} & \mathbf{9 0 0} \\ 130 & 165 & 200 & 260 & \mathbf{3 3 6} & \mathbf{4 3 2} & \mathbf{5 6 0} & \mathbf{7 3 0} & \mathbf{9 3 0} \\ & & & & & & & & \mathbf{9 6 0}\end{array}$

To reduce the error to a maximum of $\cdot 00005$, we require about 
the number of entries following : that is, about 100 entries against about 60 .

$\begin{array}{lllllllll}103 & 133 & 175 & 235 & 295 & 325 & 440 & 590 & 800 \\ 106 & 136 & 180 & 240 & 300 & 330 & 450 & 600 & 820 \\ 109 & 140 & 185 & 245 & 305 & 340 & 460 & 615 & 840 \\ 110 & 144 & 190 & 250 & 310 & 350 & 470 & 630 & 860 \\ 113 & 148 & 195 & 255 & 315 & 360 & 480 & 645 & 880 \\ 116 & 150 & 200 & 260 & 320 & 370 & 490 & 660 & 900 \\ 119 & 154 & 205 & 265 & 325 & 380 & 500 & 680 & 920 \\ 120 & 158 & 210 & 270 & 300 & 390 & 515 & 700 & 940 \\ 123 & 160 & 215 & 275 & 305 & 400 & 530 & 720 & 960 \\ 126 & 164 & 220 & 280 & 310 & 410 & 545 & 740 & 980 \\ 129 & 168 & 225 & 285 & 315 & 420 & 560 & 760 & \\ 130 & 170 & 230 & 290 & 320 & 430 & 575 & 780 & \end{array}$

In each case the smallest number of entries has not been taken but a series of numbers has been arranged so as to make the use of the proposed table rapid and simple. The smallest number of entries is given for the four figure table correct to 0001 by the numbers

$\begin{array}{lllllllll}104 & 131 & 167 & 212 & 272 & 348 & 447 & 576 & 743 \\ 108 & 136 & 174 & 221 & 283 & 363 & 466 & 601 & \mathbf{7 7 5} \\ 112 & 142 & 181 & 230 & 295 & 378 & 486 & 627 & 809 \\ 116 & 148 & 188 & 240 & 307 & 394 & 507 & 654 & 844 \\ 121 & 154 & 196 & 250 & 320 & 411 & 529 & 682 & 881 \\ 126 & 160 & 204 & 261 & 334 & 429 & 552 & 712 & 919 \\ & & & & & & & & 959\end{array}$

or 55 entries in all; but it is clear that these entries are not so convenient as the somewhat larger number selected above.

\section{On the Contact-Property of the Eleven-point Conic.}

By R. E. Allardice, M.A.

It is sometimes the case that geometrical theorems, which are usually enunciated as properties of the triangle or the quadrilateral, may be stated more succinctly, and in a form that better suggests generalisation, as properties of the complete quadrilateral. Thus 